PHYSICAL REVIEW B 92, 245436 (2015)

\title{
Quantum fluctuations stabilize skyrmion textures
}

\author{
A. Roldán-Molina \\ Instituto de Física, Pontificia Universidad Católica de Valparaíso, Avenida Universidad 330, Curauma, Valparaíso, Chile \\ M. J. Santander \\ Departamento de Física, Universidad de Santiago de Chile and CEDENNA, Avda. Ecuador 3493, Santiago, Chile \\ A. S. Nunez \\ Departamento de Física, Facultad de Ciencias Físicas y Matemáticas, Universidad de Chile, Casilla 487-3, Santiago, Chile
}

\author{
J. Fernández-Rossier*
}

International Iberian Nanotechnology Laboratory, Av. Mestre Jose Veiga, 4715-310 Braga, Portugal

(Received 26 January 2015; revised manuscript received 21 October 2015; published 23 December 2015)

\begin{abstract}
We study the quantum spin waves associated to skyrmion textures. We show that the zero-point energy associated to the quantum spin fluctuations of a noncollinear spin texture produce Casimir-like magnetic fields. We study the effect of these Casimir fields on the topologically protected noncollinear spin textures known as skyrmions. In a Heisenberg model with Dzyalonshinkii-Moriya interactions, chosen so the classical ground state displays skyrmion textures, we calculate the spin-wave spectrum, using the Holstein-Primakoff approximation, and the associated zero-point energy, to the lowest order in the spin-wave expansion. Our calculations are done both for the single-skyrmion case, for which we obtain a discrete set of skyrmion bound states, as well as for the skyrmion crystal, for which the resulting spectrum gives the spin-wave bands. In both cases, our calculations show that the Casimir magnetic field contributes up to $10 \%$ of the total Zeeman energy necessary to delete the skyrmion texture with an applied field.
\end{abstract}

DOI: 10.1103/PhysRevB.92.245436

PACS number(s): 75.30.Ds, 03.75.Lm, 74.50.+r

\section{INTRODUCTION}

The properties of many magnetic materials with broken spin symmetries are correctly described in terms of magnetic moments that have a well-defined orientation and are, in this sense, classical. Yet quantum fluctuations are invariably present. They arise, ultimately, from the fact that the different projections of the spin operator do not commute with each other. The standard [1,2] description of spin excitations of these broken symmetry magnetic materials in terms of quantum spin waves implies that, even in the ground state, there is a zero-point (ZP) energy ultimately associated to fluctuations of the magnetization field [1,2]. This ZP energy vanishes in the case of collinear isotropic ferromagnets but in general is nonzero.

In the case of quantum electrodynamics, the vacuum energy, $\mathcal{E}_{\mathrm{ZP}}$, associated to the zero-point fluctuations of the electromagnetic field between two parallel plates depends on the distance $L$ between them, resulting on an effective Casimir force, $F=-\frac{\delta \mathcal{E}_{\mathrm{ZP}}}{\delta L}$ [3]. In this work we show that, analogously, the vacuum energy associated to the quantum spin fluctuations of a given spin texture depends on an ensemble of classical variables encoded in the local spin orientation $\vec{\Omega}_{i}$, resulting in an effective Casimir field $\vec{h}_{\mathrm{ZP}}^{i}=-\frac{\delta \mathcal{E}_{\mathrm{ZP}}}{\delta \vec{\Omega}_{i}}$. This general feature of the quantum fluctuations is applied to study the stability of noncollinear magnetic textures such as skyrmions. We show that proper account of those fluctuations leads to a reduction in the energy of skyrmion textures, rendering them stable in

\footnotetext{
*Permanent address: Departamento de Física Aplicada, Universidad de Alicante.
}

regions where a purely classical analysis predicts them to be metastable.

Magnetic skyrmions are topologically protected spin structures [4]. Observed recently, both in chiral magnets [5-11] and in engineered surfaces [12-14], they have received attention for potential applications in spintronics because it is possible to control their position with very low current densities [15]. In addition, skyrmions display several features that are convenient from the viewpoint of potential applications, such as dimensions within the nanometric scale, topological protection, and high mobility. They have been observed in bulk magnets $\mathrm{MnSi}$ [5-7], $\mathrm{Fe}_{1-x} \mathrm{Co}_{x} \mathrm{Si}[8,9,16,17], \mathrm{Mn}_{1-x} \mathrm{Fe}_{x} \mathrm{Ge}$ [18], and FeGe [19] using neutron scattering and Lorentz transmission electron microscopy. As it was reported, in these systems an external magnetic field can induce skyrmions with diameters of about a few tens of nanometers. The inclusion of spin transfer torques, as it is shown by numerical simulations, can be used to nucleate and manipulate isolated skyrmions [20,21], and they respond by moving coherently in response to ultrasmall currents [6,20-23]. In addition, two-dimensional atomic-scale magnetic nanoskyrmion lattices have also been observed by means of spin-polarized scanning tunneling microscopy in a monoatomic layer of $\mathrm{Fe}$ atoms on top of the $\operatorname{Ir}(111)$ surface [12]. In this case, skyrmions arrays with a length scale of only $1 \mathrm{~nm}$ are stabilized by the interplay between Dzyaloshinskii-Moriya interaction and the breaking of inversion symmetry at surfaces and interfaces.

Previous work [24] has addressed the study of skyrmions spin excitations in a semiclassical approximation for rather large systems with up to $10^{5}$ localized moments. An effective theory of magnon-skyrmion scattering in a chiral magnet has been recently carried out by Schütte et al. [25]. Here we 
treat smaller skyrmions, using a complementary approach that permits us to study quantum fluctuations associated to the excitations relevant for atomic scale skyrmions [12] for which the effects of quantum fluctuations of the magnetization cannot be neglected at the outset. The problem of zeropoint energy for topological solitons has been studied in the ferromagnetic isotropic continuum model [26]. Here we study a discrete system of spins including as well as ferromagnetic exchange, uniaxial anisotropy, external magnetic field, and Dzyaloshinskii-Moriya interaction.

\section{TWO-DIMENSIONAL CHIRAL SPIN SYSTEM HAMILTONIAN}

The noncollinear spin alignment between first neighbors is promoted by the competition between the standard Heisenberg interaction $\left(-J \vec{S}_{i} \cdot \vec{S}_{j}\right)$ and the antisymmetric DzyaloshinskiiMoriya interaction (DMI) term $\vec{D}_{i, j} \cdot \vec{S}_{i} \times \vec{S}_{j}$, which is only present in noncentrosymmetric systems. In particular, this competition leads to helical spin textures with fixed chirality.

Application of a magnetic field $\mathbf{h}$ along the $\hat{z}$ direction (perpendicular to the system surface and then to $\vec{D}_{i, j}$ for any pair $i, j$ ) breaks the up-down symmetry in the system, resulting, for a range of fields, in an abrupt change in the ground state. In this manner, the external magnetic field and a uniaxial anisotropy can stabilize an isolated skyrmion on a finite ferromagnetic system with DMI. A minimal model that captures the essence of the physics just described is given by the following Hamiltonian with four contributions, the Heisenberg and DM interactions, the Zeeman term, and the uniaxial anisotropy:

$$
\begin{aligned}
H= & -\sum_{\langle i, j\rangle} J_{i, j} \vec{S}_{i} \cdot \vec{S}_{j}+\sum_{\langle i, j\rangle} \vec{D}_{i, j} \cdot\left(\vec{S}_{i} \times \vec{S}_{j}\right) \\
& -\sum_{i} \vec{h} \cdot \vec{S}_{i}-K \sum_{i}\left(S_{i}^{z}\right)^{2},
\end{aligned}
$$

where the interactions are taken with nearest neighbors only.

\section{CALCULATION OF SPIN WAVES}

\section{A. Classical ground state}

Since exact diagonalization of this Hamiltonian is not possible except for very small systems, we adopt the standard spin-wave approach, which is implemented in two clearly separated steps. First, we treat Eq. (1) as a classical functional $\mathcal{E}_{\mathrm{cl}} \equiv H\left(\vec{\Omega}_{i}\right)$, where the spin operators $\vec{S}$ are replaced by classical vectors $\vec{\Omega}_{i}$. The classical ground state, defined as the configuration $\vec{\Omega}_{i}$ that minimizes the functional $\mathcal{E}_{\mathrm{cl}}$, is determined by self-consistent iteration.

We consider two complementary situations. First, a single skyrmion system in a square lattice with up to $45 \times 45$ sites with hard boundary conditions at the edges, implemented by application of of a large magnetic field in the border sites. Second, a larger system with a $62 \times 36$ simulation cell with Bloch-like boundary conditions, is used to account for the quantum effects over a skyrmion lattice. The main results of the paper are very similar for the two geometries. Unless otherwise stated, we assume [24,27] $S=1, J=3.0 \mathrm{meV}, D=0.6 \mathrm{meV}$,
$\vec{D}_{i, j} \| \vec{r}_{i, j}$, and $K=0.5\left(D^{2} / J\right)$, where $\vec{r}_{i, j}$ is the unit vector between the sites $i$ and $j$.

For a given value of $D / J$ and $A / J$, the classical ground state can adopt three basic forms, depending on the value of the Zeeman field $h$. At zero field the ground state is an in-plane helimagnet. The pitch of the helices is the parameter $D / J$. Application of the Zeeman field results in an off-plane tilting of the spins. Above $h_{c 1} \sim 0.2\left(D^{2} / J\right)$, the ground state turns into the skyrmion lattice. The density of the skyrmions is controlled as well by the parameter $D / J$. As the applied field increases, it becomes eventually favorable to align all the spins. This ferromagnetic solution becomes the classical ground state for $h_{c 2} \sim 0.8\left(D^{2} / J\right)$. Still, the skyrmion solution is metastable, and thereby with well-defined spin-wave excitations, up to fields $h_{c^{\prime}} \sim 1.67\left(D^{2} / J\right)$. It must be noted that the values of $h_{c 1}$ and $h_{c 2}$ obtained in our simulations are in line with the variational predictions [28].

\section{B. Holstein-Primakoff representation and spin-wave Hamiltonian}

The second step permits us to compute quantum fluctuations of the classical solution. For that matter, we represent the spin operators in terms of Holstein-Primakoff (HP) bosons $[2,29]$ :

$$
\begin{aligned}
\mathbf{S}_{i} \cdot \vec{\Omega}_{i} & =S-n_{i}, \\
S_{i}^{+} & =\sqrt{2 S-n_{i}} a_{i}, \quad S_{i}^{-}=a_{i}^{\dagger} \sqrt{2 S-n_{i}},
\end{aligned}
$$

where $\vec{\Omega}_{i}$ is the spin direction of the classical ground state on the position $i, a_{i}^{\dagger}$ is a Bosonic creation operator, and $n_{i}=a_{i}^{\dagger} a_{i}$ is the boson number operator. The operator $n_{i}$ measures the deviation of the system from the classical ground state. The essence of the spin wave calculation is to represent the spin operators in Eq. (1) by the HP bosonic representation and to truncate the Hamiltonian up to quadratic order in the bosonic operators. The linear terms in the bosonic operators vanish when the expansion is done around the correct classical ground state. This approach has been widely used in the calculations of spin waves for ferromagnetic and antiferromagnetic ground states [1,2]. In the spin-wave approximation we thus approximate $S_{i}^{-}=\sqrt{2 S} a_{i}^{\dagger}$, so the creation of a HP boson is equivalent to the removal of one unit of spin angular momentum from the classical ground state.

After some algebra [30] the quadratic Hamiltonian can be reduced to a symmetric form in creation an annihilation operators written as:

$$
H=\mathcal{E}_{\mathrm{cl}}+\mathcal{E}_{0}+\sum_{i, j}\left(a_{i}^{\dagger}, a_{j}\right)\left(\begin{array}{cc}
t_{i, j} & \tau_{i, j} \\
\tau_{i, j}^{*} & t_{i, j}^{*}
\end{array}\right)\left(\begin{array}{l}
a_{j} \\
a_{j}^{\dagger}
\end{array}\right),
$$

where the constant $\mathcal{E}_{0}$ is a quantum contribution to the ground-state energy that arises naturally in this expression as a consequence of applying the bosonic conmutation relations in order to bring the Hamiltonian to its symmetric form. The second and third terms in Eq. (3) correspond to the spin-waves Hamiltonian $H_{\mathrm{SW}}$. Importantly, $\mathcal{E}_{\mathrm{cl}}, \mathcal{E}_{0}, t_{i, j}$, and $\tau_{i, j}$ are a functional of the classical ground-state configuration $\vec{\Omega}_{i}$ that satisfies $\frac{\delta \mathcal{E}_{\mathrm{cl}}}{\delta \vec{\Omega}_{j}}=0$. 
The off-diagonal terms in Eq. (3) do not conserve the number of HP bosons and are in general present for the noncollinear classical ground state $[1,2]$. In consequence, the ground state $|\mathrm{GS}\rangle$ of the Hamiltonian is not the vacuum of the $a$ bosons and, thereby, the ground-state energy at the spin-wave approximation level differs from the classical ground-state energy. The Hamiltonian $H_{\mathrm{Sw}}$ is solved by means of a paraunitary $[30,31]$ transformation, analogously to the usual Bogoliubov $u-v$ transformation for bosons [32], that leads to:

$$
H_{\mathrm{SW}}=\mathcal{E}_{0}+\sum_{\nu=1}^{N} \hbar \omega_{\nu}\left(\alpha_{\nu}^{\dagger} \alpha_{\nu}+\frac{1}{2}\right),
$$

where $N$ corresponds to the total number modes (number of sites) and $\alpha_{v}$ are bosonic operators formed by linear combination of both $a$ and $a^{\dagger}$ that annihilate spin waves with energy $\hbar \omega_{\nu}$. The corresponding state can be written down as:

$$
\left|\psi_{v}\right\rangle=\alpha_{v}^{\dagger}|\mathrm{GS}\rangle=\sum_{i}\left[v_{v}^{*}(i) a_{i}^{\dagger}+u_{v}^{*}(i) a_{i}\right]|\mathrm{GS}\rangle .
$$

Here $u(i)$ and $v(i)$ can be interpreted as the conventional and anomalous parts of the wave spin-wave function.

\section{Properties of the skyrmion magnon modes}

We apply this general formalism the case of a single skyrmion an isolated skyrmion. It must be noted that in the case of an ideal crystal, a single skyrmion cannot be a stable configuration. However, isolated skyrmions are observed with STM, perhaps stabilized by some surface imperfection [14]. In Fig. 1 we show the evolution of the four lowest energy modes as function of magnetic field for the single skyrmion case. The abrupt change at $h_{c^{\prime}}$ is a consequence of the fact that for larger fields, the skyrmion-like solution is no longer allowed and a transition to ferromagnetic order takes place. It is apparent that the skyrmion has in-gap spin-wave excitations, in line with those obtained using the semiclassical approximation [24]. The first excitation has a very low energy and can be readily associated with a gyrotropic traslation mode. In Fig. 2 we show
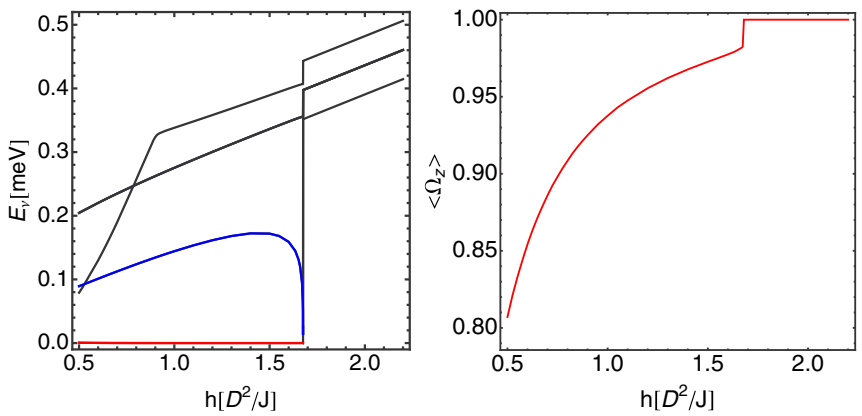

FIG. 1. (Color online) Left panel: Spin-wave spectrum as a function of magnetic field. Right panel: Average spin projection along the $z$ axis as a function of the external magnetic field. As the field grows the size of the skyrmion is reduced. At a field of $h \sim 1.67\left(D^{2} / J\right)$ the system can no longer sustain the skyrmion texture and a transition towards a ferromagnetic state is induced.
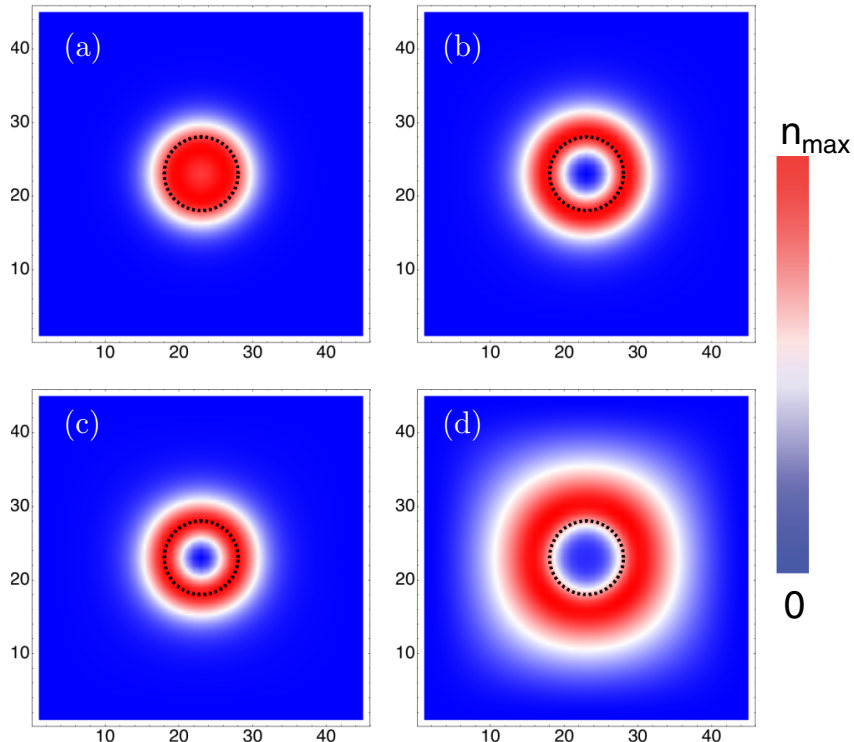

FIG. 2. (Color online) Magnonic occupation of the four lowest energy excited states displayed in (a), (b), (c), and (d) respectively. The plots correspond to $D=0.2 J$ and a field $h=0.75\left(D^{2} / J\right)$. On each figure the dashed line corresponds to the perimeter of the skyrmion. The maximum values of the occupation for each excited state are $n_{\max }=7.1 \times 10^{-3} ; 5.6 \times 10^{-3} ; 4.8 \times 10^{-3}$ and $2.1 \times 10^{-3}$ respectively.

the magnonic occupation, defined as

$$
n_{v}(i)=\left\langle\psi_{\nu}\left|a_{i}^{\dagger} a_{i}\right| \psi_{\nu}\right\rangle
$$

for the four lowest energy modes, where $\left|\psi_{\nu}\right\rangle \equiv \alpha_{\nu}^{\dagger}|\mathrm{GS}\rangle$.

Our approach allows us to calculate all the spin-wave excitations of the classical ground state. In the case of a skyrmion embedded in a ferromagnetic background, this includes both modes localized in the skyrmion, or skyrmion magnon modes, as well as spin waves that dwell the collinear region, or bulk magnon modes. We find that the skyrmion magnon modes have lower energy and thereby are more relevant. In order to describe their physical properties we calculate the magnon occupation and the space distribution of both real and imaginary parts of the quantity

$$
\mathcal{S}_{\nu}(i) \equiv\left\langle\mathrm{GS}\left|a_{i} \alpha_{\nu}^{\dagger}\right| \mathrm{GS}\right\rangle
$$

that describes the overlap between the spin-wave function $v$ and the state where a spin-flip excitation is created at atom $i$. Inspection of the four lowest energy excited states, shown in Fig. 2, shows that they are modes confined around the skyrmion, as opposed to extended spin-wave modes. Additional insight of their nature comes from inspection of the $\operatorname{Re}(\mathcal{S})$ and $\operatorname{Im}(\mathcal{S})$, shown in Fig. 3. In particular, the first and third modes look like modes with the $x+i y$ and $x$ - iy symmetry, where $x$ and $y$ are defined with respect to the diagonals in the figure. On the same vein, the fourth mode has a symmetry $x^{2}-y^{2}+i x y$. Thus, these modes have a well-defined orbital angular momentum that is compatible with rotation modes of the skyrmion.

The skyrmion-ferromagnet transition is driven by a reduction of the energy of the breathing mode. The abrupt reduction of the second excitation energy near the critical field signals 

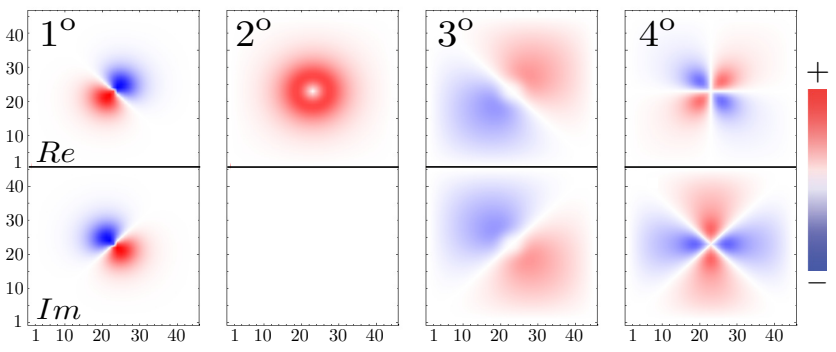

FIG. 3. (Color online) Real and imaginary part of the quantity $\left\langle\mathrm{GS}\left|a_{i} \alpha_{v}^{\dagger}\right| \mathrm{GS}\right\rangle$ for the four lowest energy excited states. The plots correspond to $D=0.2 \mathrm{~J}$ and a field $h=0.75\left(D^{2} / J\right)$.

the instability of the skyrmion texture. Below the critical field, both internal and extended spin-wave states are present in the system. For fields larger than the critical field the spin-wave spectrum correspond to the usual ferromagnetic spin waves.

\section{ZERO-POINT MAGNON ENERGY}

We now discuss the main result of the manuscript. In contrast with the semiclassical theory, the quantum theory of spin waves also yields a renormalization of the energy of the ground state whose wave function is, by definition, the vacuum of the $\alpha$ operators, $\alpha_{\nu}|\mathrm{GS}\rangle=0$. In the case of noncollinear classical order, the ground state differs from the vacuum of HP bosons. The ground-state energy reads:

$$
\mathcal{E}_{\mathrm{GS}}=\mathcal{E}_{\mathrm{cl}}+\mathcal{E}_{0}+\frac{1}{2} \sum_{\nu=1}^{N} \hbar \omega_{v} \equiv \mathcal{E}_{\mathrm{cl}}+\mathcal{E}_{\mathrm{ZP}},
$$

which differs from the classical energy due to the contribution coming from quantum fluctuations, or zero-point energy, $\mathcal{E}_{\mathrm{ZP}} \equiv \mathcal{E}_{\mathrm{GS}}-\mathcal{E}_{\mathrm{cl}}$. In the case of ferromagnetic solutions on a pure uniaxial system, it turns out that $\mathcal{E}_{\text {ZP }}$ vanishes $[1,2]$ because the collinear ferromagnetic ground state is also an eigenstate of the exact Hamiltonian.

In general, the vacuum energy $\mathcal{E}_{\mathrm{ZP}}$ is not zero and, as it turns out, it contributes significantly to the relative stability of different spin configurations.

\section{A. Zero-point energy of an isolated skyrmion}

As a first application of the concept, we study the case of a single skyrmion. In Fig. 4 we compare the energy difference between the skyrmion and the collinear configuration, as a function of the applied field $h$, calculated at the classical level (blue line) and including $\mathcal{E}_{\mathrm{ZP}}$ (red line) for the single skyrmion geometry. We have studied three cases: $D=0.2 \mathrm{~J}, D=0.3 \mathrm{~J}$, and $D=0.4 J$ which show very similar behaviors. As in the case of the Belavin-Polyakov soliton [26], the $\mathcal{E}_{\text {ZP }}$ reduces the classical energy of the skyrmion, increasing by $10 \%$ the critical field $h_{c}$ above which the skyrmion configuration passes to the metastable regime.

\section{B. Casimir spin torque}

By analogy with the Casimir effect, variations of the vacuum energy with the local moment orientation can be associated with an effective Casimir magnetic field. This
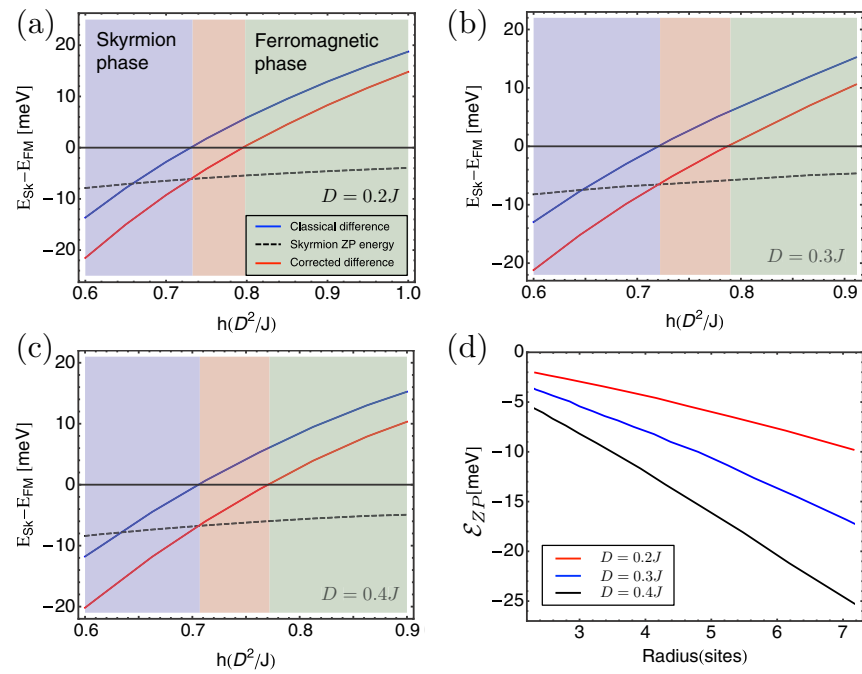

FIG. 4. (Color online) Stability energy for skyrmions at three different values of $D=0.2 J$ (a), $0.3 J$ (b), $0.4 J$ (c) and zero-point energy as function of the skyrmion radius (d). In each case we have taken $K=0.5 D^{2} / J$. The crossing between the curves and the horizontal axis (a)-(c) sets the boundary for the region of stability for the skyrmion in classical and corrected approaches (blue and red lines respectively). Corrected energy difference including the zero-point fluctuations, enlarging the region of stability for skyrmions.

Casimir field is the magnetic analog of the Casimir force [35]. In this manner, the effective field on a given spin can be defined as:

$$
\vec{h}_{i}=-\frac{\delta \mathcal{E}_{\mathrm{GS}}}{\delta \vec{\Omega}_{i}}=-\frac{\delta}{\delta \vec{\Omega}_{i}}\left(\mathcal{E}_{\mathrm{cl}}+\left\langle\mathrm{GS}\left|\mathcal{H}_{\mathrm{SW}}\right| \mathrm{GS}\right\rangle\right)
$$

The first contribution is the classical field, which vanishes identically by construction. Using this and the HellmanFeynman theorem [33], the Casimir field $\vec{h}_{\mathrm{ZP}}$ read:

$$
\vec{h}_{\mathrm{ZP}}^{i}=-\left\langle\mathrm{GS}\left|\frac{\delta \mathcal{H}_{\mathrm{SW}}}{\delta \vec{\Omega}_{i}}\right| \mathrm{GS}\right\rangle
$$

and they can be readily calculated. Our results for the Casimir field are displayed in Fig. 5, together with the projection of the classical magnetization over the $z$ axis (top panel), and the expectation value, computed with the ground-state wave function, of the magnon occupation. For reference, we sketch the spherical coordinate system as shown in the inset of Fig. 5(b). At the center of the skymion the magnetization points along $-\hat{z}$, opposite from the magnetization of the surroundings, as can be seen in Fig. 5(a).

The stabilization of the skyrmion configuration with respect to the applied field can be associated to the Casimir field using the following argument. The Zeeman contribution enters the energy of the system as $-\Omega_{z} h$; in the same manner, the contribution of the Casimir field in the classical direction $\vec{\Omega}$ corresponds to $-S\left(\vec{h}_{\mathrm{ZP}} \cdot \vec{\Omega}\right)=-S h_{\Omega}$. In Fig. 5(c) we plot $h_{\Omega}$, it is clear from here that the contribution of the Casimir field on the classical direction is to lower the energy of the skyrmion state.

In addition to the stabilization of the skyrmion solution, quantum fluctuations can have other observable consequences that we discuss now. In order to characterize the density of 


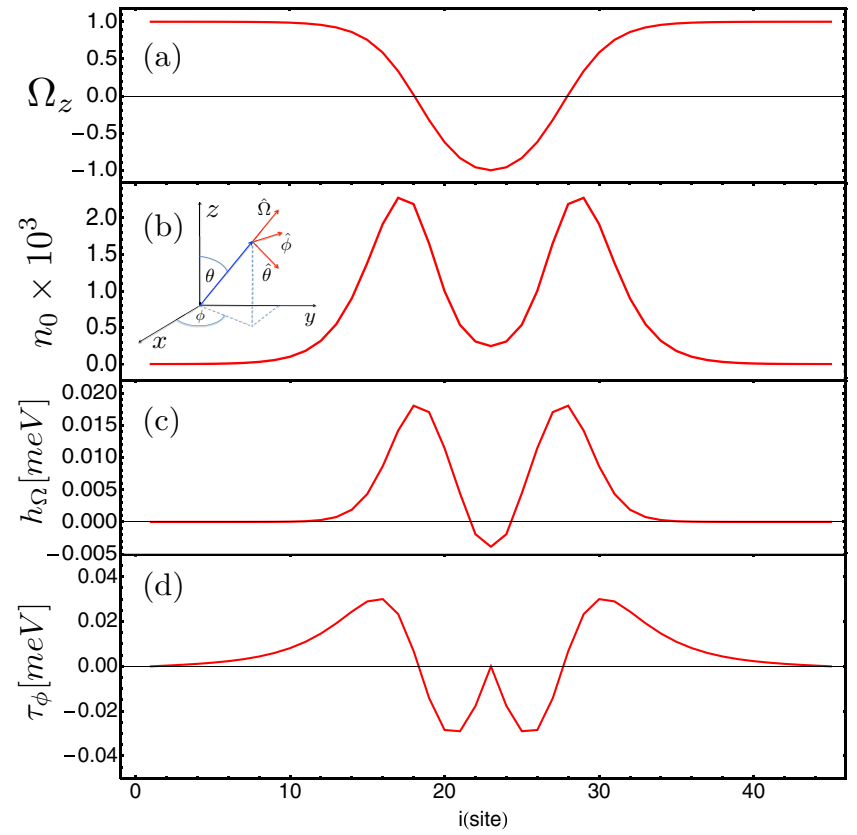

FIG. 5. (Color online) Magnetization profile on $z$ direction (a), magnonic occupation (b), and Casimir field ( $\Omega$ direction) (c), and Casimir torque ( $\phi$ direction) (d) on a skyrmion ground state for $D=$ $0.2 J$ and $h=0.75\left(D^{2} / J\right)$. The local coordinate system for spins and the corresponding Casimir fields is shown in the inset in panel (b). As consequence of the Casimir field on direction $\Omega$, the skyrmion zeropoint energy is reduced, and thereby it increase the texture stability.

spin fluctuations of a given eigenstate of Hamiltonian (4), $\left|\psi_{\nu}\right\rangle \equiv \alpha_{\nu}^{\dagger}|\mathrm{GS}\rangle$, we have defined the magnonic occupation in Eq. (6). Importantly, this quantity is nonzero even in the ground state, $n_{0}^{i}=\left\langle\mathrm{GS}\left|a_{i}^{\dagger} a_{i}\right| \mathrm{GS}\right\rangle$, reflecting the zero-point quantum fluctuations that are a consequence of a noncollinear classical state. The zero-point magnonic occupation of the skyrmion ground state is shown in Fig. 5(b). Calculations using different values for $D, J$, and $K$ show that the magnitude of the ZP fluctuations increases as the size of the skyrmion is reduced. The ZP fluctuations have observable consequences. Since $\left\langle\vec{S} \cdot \Omega_{i}\right\rangle=S-\left\langle a_{i}^{\dagger} a_{i}\right\rangle$, for $S>1 / 2$ both the zero-point motion and the thermally excited spin waves will reduce the effective atomic magnetic moments of skyrmion solutions, an effect that should be observable with local probes, such as STM.

Importantly, the Casimir magnetic field exert torque on the magnetic moments, the Casimir spin torque, defined as $\vec{\tau}_{i}=$ $\vec{\Omega}_{i} \times \vec{h}_{\mathrm{ZP}}^{i}$. The Casimir spin torque on the skyrmion is shown in Fig. 4(d) and dependence of maximum torque with the external field for different anisotropy constant $K$ is shown in Fig. 6. In the Fig. 6 we can see that both the Casimir torque as $\mathcal{E}_{\mathrm{ZP}}$ depends significantly on the uniaxial anisotropy constant $K$. This dependence comes from the misalignment between local spins and anisotropy easy axis. The ZP energy, and thereby the ZP Casimir spin torques, depend on the energy of the spin-wave excitations, which are interesting on their own right and could be observed by means of inelastic electron tunneling spectroscopy, as recently demonstrated for spin waves in short ferromagnetic chains [34].
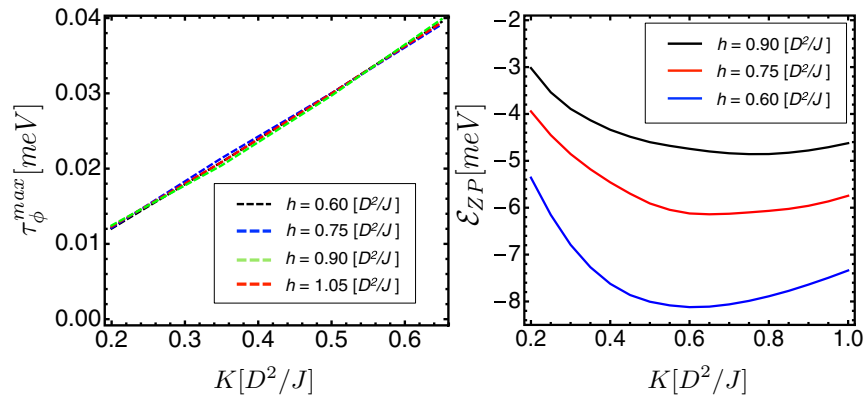

FIG. 6. (Color online) Maximum Casimir torque on $\phi$ direction (left panel) and zero-point energy (right panel) for the single skyrmion geometry as function of anisotropy constant $K$ and different fields.

\section{SKYRMION LATTICE}

We now consider the case of a skyrmion crystal. In order to estimate the effect of the ZPE on a skyrmion lattice we consider a larger system and impose Bloch-like boundary conditions over the magnon modes. In Fig. 7 inset we show the unit cell $(62 \times 36$ sites $)$ taken for the skyrmion lattice calculations for $D=0.2 \mathrm{~J}$. Just as before we begin by solving the classical problem. In this case the classical solution is found imposing periodic boundary conditions. The dimensions of our simulation cell have been chosen to fit two skyrmions as shown in Fig. 7 (inset).

As a result of the periodic boundary conditions, we can classify the spin-wave states using Bloch theorem, so we assign them a crystal momentum $\vec{k}$ and a band index. In this representation, for every point $\vec{k}$ in the first Brillouin we have

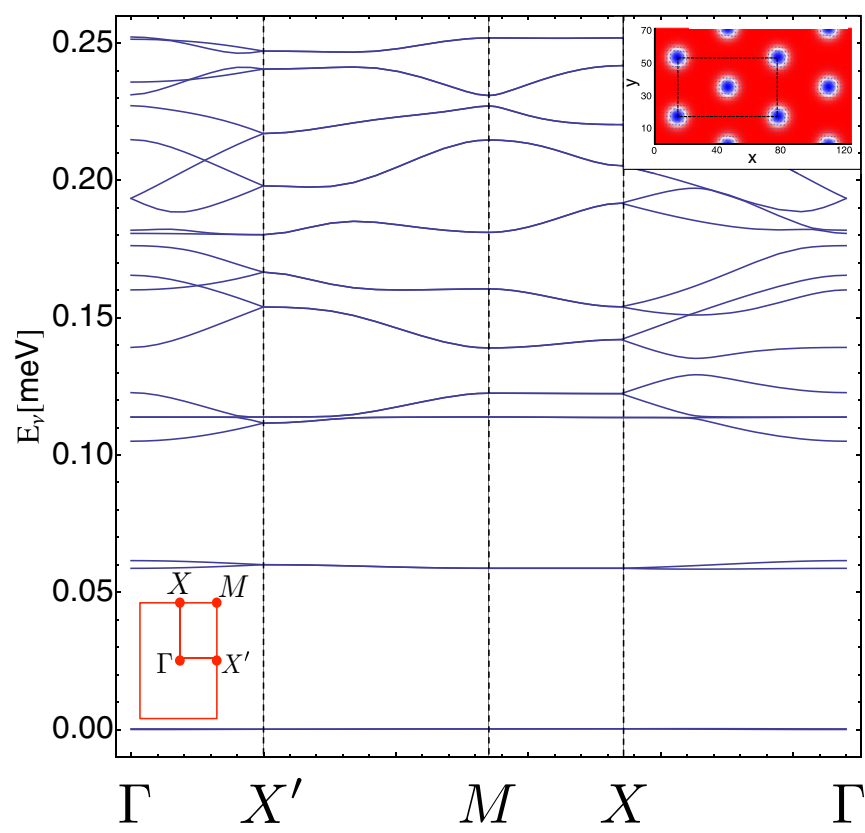

FIG. 7. (Color online) Spin-wave bands calculated for the skyrmion crystal considering a two-skyrmions unit cell (see inset, upper right). Bands are shown along the high symmetry lines in the Brillouin zone, shown in the inset (lower left). The narrow bandwidth reflect the weak interskyrmion coupling. Flat bands correspond to localized modes. 

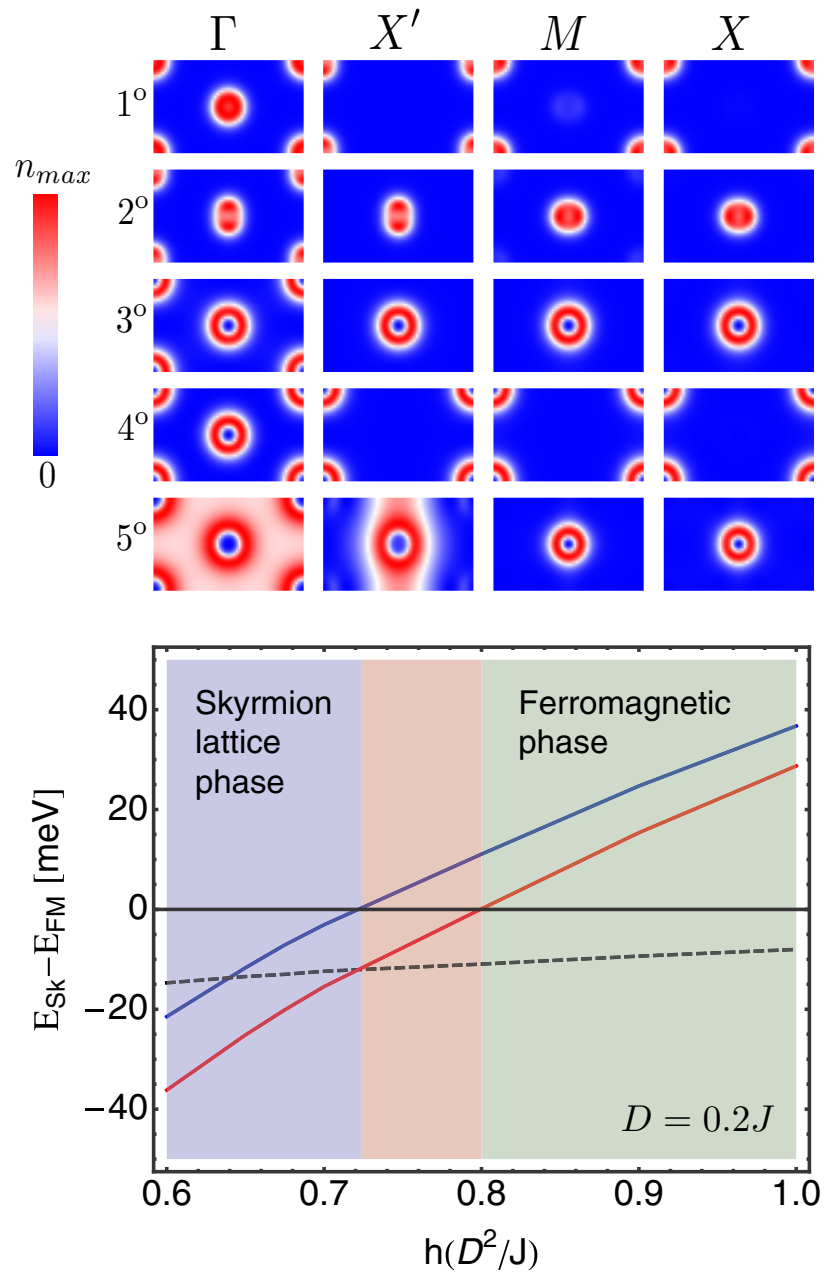

FIG. 8. (Color online) Top: Magnonic occupation, $n_{i}=\left\langle\psi_{\nu}\left|a_{i}^{\dagger} a_{i}\right| \psi_{\nu}\right\rangle$, for the lowest-lying bands at four characteristic points in the Brillouin zone $\left(\Gamma, X^{\prime}, M\right.$, and $\left.X\right)$. The localization of the spin waves around the skyrmion is apparent for the first four bands. In contrast, higher-energy bands correspond to extended modes. Bottom: Same as in Fig. 4 with the classical and zero-point energies evaluated over the skyrmion lattice.

a spin-wave Hamiltonian $H(\vec{k})$ for which the entire formalism described in the previous section is valid. This permits us to obtain the magnon energy bands, $\omega_{\mu}(\vec{k})$, shown in Fig. 7. It is apparent that the lowest energy bands are separated by band gaps and have a very small dispersion, as expected from the fact that for a single skyrmion the lowest energy spin waves are localized modes.
The degree of localization of the different spin-wave modes is quantified by plotting the magnon density for various bands, evaluated at the high symmetry points of the Brillouin zone, shown in Fig. 8. Thus, the lowest lying bands are flat bands associated with the translational and breathing modes of the isolated skyrmion.

Straightforward application of Eq. (8) including a sum over the momentum index, permits us to calculate the zero-point energy contribution for the skyrmion crystal, resulting in the energy difference between the collinear and skyrmion lattice configurations. The results, measured in energy per unit cell, are depicted in Fig. 8 (bottom). We see that the main conclusions drawn from the isolated skyrmion calculation can be extended, even with quantitative accuracy, to the skyrmion lattice ground state. This calculation confirms the validity of the single skyrmion calculation discussed in the previous section.

\section{SUMMARY AND CONCLUSIONS}

In summary, we have applied the Holstein-Primakoff method to obtain a quantum theory for spin waves associated to skyrmions. This quantum theory of spin waves permits us to calculate the observable consequences of quantum spin fluctuations in the energetic balance of skyrmions-like textures. We have found that the zero-point energy benefits the skyrmion stability, increasing significantly the critical field necessary to erase the skyrmion. We show that this stabilization can be associated to a Casimir magnetic field driven by zero-point fluctuations analogous to the Casimir effect. We have also found that zero-point fluctuations renormalize the shape of the skyrmion and shrink the atomic magnetization, an effect that becomes significant for small skyrmions and could be observed by means of spin-polarized scanning tunneling microscopy. We have applied our methodology both to isolated skyrmions and skyrmion crystals and have obtained consistent results.

\section{ACKNOWLEDGMENTS}

The authors acknowledge funding from Fondecyt Grant No. 1150072, Grant No. ICM P10-061-F by Fondo de Innovación para la Competitividad-MINECON, and Anillo Grant No. ACT 1117. A.S.N. also acknowledges support from Financiamiento Basal para Centros Científicos y Tecnológicos de Excelencia, under Project No. FB 0807(Chile). A.R.M., M.J.S., and A.S.N. acknowledge the hospitality of INL.
[1] K. Yosida, Theory of Magnetism (Springer, Heidelberg 1996).

[2] Assa Auerbach, Interacting Electrons and Quantum Magnetism (Springer, New York 1994).

[3] H. B. G. Casimir, Proc. K. Ned. Akad. Wet. 51, 793 (1948).

[4] U. Al Khawaja and H. T. C. Stoof, Nature 411, 918 (2001); J. Armaitis, H. T. C. Stoof, and R. A. Duine, Phys. Rev. Lett. 110, 260404 (2013).
[5] S. Muhlbauer, B. Binz, F. Jonietz, C. Pfleiderer, A. Rosch, A. Neubauer, R. Georgii, and P. Boni, Science 323, 915 (2009).

[6] F. Jonietz, S. Muhlbauer, C. Pfleiderer, A. Neubauer, W. Munzer, A. Bauer, T. Adams, R. Georgii, P. Boni, R. A. Duine, K. Everschor, M. Garst, and A. Rosch, Science 330, 1648 (2010).

[7] A. Neubauer, C. Pfleiderer, B. Binz, A. Rosch, R. Ritz, P. G. Niklowitz, and P. Boni, Phys. Rev. Lett. 102, 186602 (2009). 
[8] W. Munzer, A. Neubauer, T. Adams, S. Muhlbauer, C. Franz, F. Jonietz, R. Georgii, P. Boni, B. Pedersen, M. Schmidt, A. Rosch, and C. Pfleiderer, Phys. Rev. B 81, 041203(R) (2010).

[9] P. Milde, D. Kuhler, J. Seidel, L. M. Eng, A. Bauer, A. Chacon, J. Kindervater, S. Muhlbauer, C. Pfleiderer, S. Buhrandt, C. Schutte, and A. Rosch, Science 340, 1076 (2013).

[10] M. Nagao, Y. So, H. Yoshida, M. Isobe, T. Hara, K. Ishizuka, and K. Kimoto, Nat. Nanotechnol. 8, 325 (2013).

[11] S. Seki, X. Z. Yu, S. Ishiwata, and Y. Tokura, Science 336, 198 (2012).

[12] S. Heinze, K. von Bergmann, M. Menzel, J. Brede, A. Kubetzka, R. Wiesendanger, G. Bihlmayer, and S. Blugel, Nat. Phys. 7, 713 (2011).

[13] N. Romming, C. Hanneken, M. Menzel, J. E. Bickel, B. Wolter, K. von Bergmann, A. Kubetzka, and R. Wiesendanger, Science 341, 636 (2013)

[14] N. Romming, A. Kubetzka, C. Hanneken, K. von Bergmann, and R. Wiesendanger, Phys. Rev. Lett. 114, 177203 (2015)

[15] A. Fert, V. Cros, and J. Sampaio, Nat. Nanotechnol. 8, 152 (2013).

[16] C. Pfleiderer et al., J. Phys.: Condens. Matter 22, 164207 (2010).

[17] X. Z. Yu, Y. Onose, N. Kanazawa, J. H. Park, J. H. Han, Y. Matsui, N. Nagaosa, and Y. Tokura, Nat. Mater. 465, 901 (2010).

[18] K. Shibata, X. Z. Yu, T. Hara, D. Morikawa, N. Kanazawa, K. Kimoto, S. Ishiwata, Y. Matsui, and Y. Tokura, Nat. Nanotechnol. 8, 723 (2013).

[19] X. Z. Yu, N. Kanazawa, Y. Onose, K. Kimoto, W. Z. Zhang, S. Ishiwata, Y. Matsui, and Y. Tokura, Nat. Mater. 10, 106 (2011).
[20] J. Iwasaki, M. Mochizuki, and N. Nagaosa, Nat. Commun. 4, 1463 (2013).

[21] J. Iwasaki, M. Mochizuki, and N. Nagaosa, Nat. Nanotechnol. 8, 742 (2013).

[22] R. E. Troncoso and A. S. Nunez, Phys. Rev. B 89, 224403 (2014),

[23] R. E. Troncoso and A. S. Nunez, Ann. Phys. 351, 850 (2014).

[24] S.-Z. Lin, C. D. Batista, and A. Saxena, Phys. Rev. B 89, 024415 (2014).

[25] C. Schütte and M. Garst, Phys. Rev. B 90, 094423 (2014).

[26] Boris A. Ivanov, Denis D. Sheka, Vasyl V. Krivonos, and Franz G. Mertens, Phys. Rev. B 75, 132401 (2007).

[27] J. Zang, M. Mostovoy, J. H. Han, and N. Nagaosa, Phys. Rev. Lett. 107, 136804 (2011).

[28] Jung Hoon Han, Jiadong Zang, Zhihua Yang, Jin-Hong Park, and Naoto Nagaosa, Phys. Rev. B 82, 094429 (2010)

[29] T. Holstein and H. Primakoff, Phys. Rev. 58, 1098 (1940).

[30] A. Roldán-Molina, M. J. Santander, A. S. Núñez, and J. Fernández-Rossier, Phys. Rev. B 89, 054403 (2014)

[31] J. H. P. Colpa, Physica A 93, 327 (1978).

[32] N. N. Bogoliubov, Bull. Acad. Sci. USSR, Phys. 11, 77 (1947); J. Phys. 9, 23 (1947).

[33] R. P. Feynman, Phys. Rev. 56, 340 (1939)

[34] A. Spinelli, B. Bryant, F. Delgado, J. Fernández-Rossier, and A. F. Otte, Nat. Mater. 13, 782 (2014)

[35] After the first version of this work was posted in the arXiv, the concept of Casimir spin torque was independently proposed by Du et al., Phys. Rev. B 92, 214409 (2015) in the context of quantum antiferromagnets. 